\title{
Effect of prior receipt of antibiotics on the pathogen distribution and antibiotic resistance profile of key Gram-negative pathogens among patients with hospital- onset urinary tract infections
}

\author{
Monique R. Bidell', Melissa Palchak Opraseuth², Min Yoon², John Mohr² and Thomas P. Lodise ${ }^{\text {1* }}$
}

\begin{abstract}
Background: This retrospective cohort study characterized the impact of prior antibiotic exposure on distribution and nonsusceptibility profiles of Gram-negative pathogens causing hospital-onset urinary tract infections (UTI).

Methods: Hospital patients with positive urine culture for Pseudomonas aeruginosa, Escherichia coli, Klebsiella pneumoniae, and other Enterobacteriaceae $\geq 3$ days after hospital admission were included. Assessment outcomes included the distribution of bacteria in urine cultures, antibiotic susceptibility patterns, and the effect of prior antibiotic exposure, defined as 0,1 , or $\geq 2$ prior antibiotics, on the distribution and antibiotic susceptibility profiles of the Gram-negative organisms.

Results: The most commonly isolated pathogens from 5574 unique UTI episodes (2027 with and 3547 without prior antibiotic exposure) were E. coli (49.5\%), K. pneumoniae (17.1\%), and P. aeruginosa (8.2\%). P. aeruginosa was significantly more commonly isolated in patients with $\geq 2$ prior antibiotic exposures (12.6\%) compared with no exposure (8.2\%; $p=0$. 036) or 1 prior exposure (7.9\%; $p=0.025)$. Two or more prior antibiotic exposures were associated with slightly higher incidences of fluoroquinolone nonsusceptibility, multidrug resistance, and extended-spectrum $\beta$-lactamase phenotype compared with 0 or 1 exposure, suggesting an increased risk for resistant Gram-negative pathogens among hospital patients with urinary tract infections occurring $\geq 3$ days after admission.
\end{abstract}

Conclusions: Clinicians should critically assess prior antibiotic exposure when selecting empirical therapy for patients with hospital-onset urinary tract infections caused by Gram-negative pathogens.

Keywords: Antibiotic use, Antimicrobial resistance, Fluoroquinolones, Gram-negative pathogens, Multidrug resistant, Urinary tract infection

\section{Background}

Increasing antibiotic resistance among Gram-negative pathogens, particularly in the hospital setting, is well documented and constitutes a major public health concern $[1,2]$. Gram-negative organisms are implicated in a number of hospital-acquired infections, with urinary tract infections (UTIs) particularly common [1, 3]. Antibiotic resistance among hospital-acquired infections owing to Gram-negative

\footnotetext{
* Correspondence: thomas.lodise@acphs.edu

'Albany College of Pharmacy and Health Sciences, 106 New Scotland

Avenue, Albany 12208-3492, NY, USA

Full list of author information is available at the end of the article
}

pathogens greatly complicates the administration of timely and appropriate therapy, placing patients at increased risk for deleterious outcomes [4-6]. Resources such as antibiograms can facilitate the empirical antimicrobial selection process [7] by characterizing local resistance patterns. However, reliance on a single collective isolate institutional antibiogram for empirical antibiotic selection is associated with several notable shortcomings. Collective isolate antibiograms developed by many institutions do not capture the distribution of pathogens associated with a particular infection, nor do they reflect the susceptibility profile of a particular pathogen at an infection site (eg, urinary tract). 
Although culture site-specific antibiograms can ameliorate this issue, they do not indicate patient-specific factors that increase the likelihood of an antibiotic-resistant infection [7].

Two critical patient characteristics that modify the risk for antibiotic-resistant infection are recent antibiotic exposure and recent admission to, or current residence in, a health care institution $[2,8,9]$. Considering the limitations associated with an antibiogram-only approach to empirical antibiotic selection, this study was designed to characterize the impact of prior antibiotic exposure on the distribution and nonsusceptibility profiles of key Gram-negative pathogens among US inpatients with hospital-onset UTIs. We specifically focused on this single modifiable risk factor because it is readily identifiable and accessible in the medical record system for all patients. Other possible patient risk factors were not included in the analysis so that a simple and straightforward guide to empirical antibiotic selection could be created.

\section{Methods}

\section{Study design and population}

This retrospective, observational study used hospital discharge data from the Premier Healthcare Database, which at the time of this study contained data from more than 435 million patient encounters. Laboratory results were available from a subset of approximately 160 facilities in the Premier Healthcare Database. Patients were included in the study if all the following criteria were met: inpatient discharge between January 1, 2012, and March 31, 2013; positive urine culture for any of the prespecified Gram-negative bacteria $\geq 3$ days after hospital admission; and receipt of an antibiotic with activity against Gram-negative pathogens on the index culture date or within the 3-day period thereafter. The first documented urine culture was included in the analysis. Duplicate isolates from subsequent urine cultures within 30 days were excluded. Duplicate isolates recovered from urine cultures $>30$ days after the index culture were included because we felt these to be representative of either recurrent or new infection. As such, we felt it was important to include these subsequent occurrences as unique episodes within the study.

The Gram-negative organisms of interest were Escherichia coli, Klebsiella pneumoniae, Pseudomonas aerugi$n o s a$, and other members of the Enterobacteriaceae family (excluding E. coli and K. pneumoniae). Prior antibiotic exposure was defined as administration of $\geq 1$ prespecified agents with Gram-negative activity during the current hospitalization and before the index urine culture. Antibiotics included for assessment of prior exposure were those available in the working Premier data set: meropenem, doripenem, imipenem, ertapenem, piperacillin/tazobactam, cefepime, ceftazidime, ceftriaxone, cefotaxime, ciprofloxacin, levofloxacin, gentamicin, tobramycin, amikacin, ampicillin/sulbactam, cefazolin, gatifloxacin, cefazolin, tigecycline, and ticarcillin/clavulanic acid (or clavulanate). Data for other groups of antibiotics, including second-generation cephalosporins and trimethoprim/sulfamethoxazole, were not available.

\section{Outcomes}

The first outcome measure was distribution and antibiotic susceptibility patterns among the prespecified Gram-negative organisms for patients meeting inclusion criteria. The second outcome measure was the association of prior antibiotic exposure, defined as 0,1 , or $\geq 2$ prior exposures, with the distribution and antibiotic susceptibility profiles of the Gram-negative organisms of interest. Organisms were assessed for susceptibility to fluoroquinolones (ciprofloxacin, levofloxacin, gatifloxacin), carbapenems (meropenem, imipenem, doripenem, ertapenem), and piperacillin/tazobactam.

For the purposes of this analysis, antibiotic susceptibility was classified as susceptible versus nonsusceptible (ie, intermediate or resistant), based on the microbiology report. Multidrug resistance was defined as nonsusceptibility to $\geq 1$ agents in $\geq 3$ antibiotic classes [10]. For $P$. aeruginosa, only meropenem-, imipenem-, or doripenem-nonsusceptibility was used to define carbapenem resistance; $P$. aeruginosa isolates were considered nonsusceptible to third-generation cephalosporins if they were resistant to ceftazidime, or, if data were unavailable, to cefepime. For Enterobacteriaceae, isolates were considered nonsusceptible to a third-generation cephalosporin if they had documented resistance to at least two agents (ceftriaxone, ceftazidime, or cefotaxime). Nonsusceptibility to third-generation cephalosporins was considered indicative of an extended-spectrum beta-lacatmase (ESBL) phenotype. For patients with missing carbapenem, piperacillin/tazobactam, or MDR susceptibility data, isolates were considered carbapenem-susceptible, piperacillin/tazobactam-susceptible, or non-MDR if they were susceptible to a third-generation cephalosporin. Otherwise, carbapenem, piperacillin/tazobactam, and MDR susceptibility data were considered missing and were not included in the analyses that evaluated the relationship between prior antibiotic exposures and antibiotic susceptibility profiles.

\section{Statistical analysis}

Unadjusted descriptive analysis was used to characterize the distribution and antibiotic susceptibility patterns of the Gram-negative organisms of interest and the effect of prior antibiotic exposure on each of these patterns. Although the study was not powered for prespecified statistical analyses, possible significance between groups $(\geq 2$ 
prior exposures compared with 0 or 1 prior exposure) was calculated using the method of Miettinen and Nurminen.

\section{Results}

Overall pathogen distribution and resistance profile

Descriptive statistics of patient demographics and characteristics of hospital visits are provided (see Additional file 1). A total of 5574 unique UTI episodes were included in the analysis $(n=2027$ prior antibiotic exposure; $n=3547$ no prior exposure), from which 6093 pathogens were isolated $(n=2227$ from patients with prior antibiotic exposure; $n=3866$ from patients with no prior exposure). The most commonly isolated pathogens were $E$. coli $(n=$ 3013; 49.5\%), K. pneumoniae ( $n=1039 ; 17.1 \%)$, and $P$. aeruginosa ( $n=502 ; 8.2 \%)$ (Fig. 1$)$. Among all pathogens, fluoroquinolone nonsusceptibility and multidrug resistance exceeded 19 and 21\%, respectively (Fig. 2). The ESBL phenotype was noted in $4.1 \%$ of pathogens, and carbapenem nonsusceptibility was noted in $<2 \%$.

\section{Effect of prior antibiotic exposure}

The distribution of pathogens in relation to prior antibiotic exposure is shown in Fig. 1. P. aeruginosa was isolated significantly more often in patients with $\geq 2$ prior antibiotic exposures $(12.6 \%)$ than in patients with no prior antibiotic exposure (8.2\%; $95 \%$ confidence intervals [CI] $0.2,10.1 ; p=0.036)$ or with 1 prior exposure $(7.9 \%$; $95 \%$ CI $0.5,10.5 ; p=0.025)$. For other pathogen groups, the presence or absence of prior antibiotic exposure did not substantially affect distribution trends, and differences were nonsignificant.

Nonsusceptibility characteristics with respect to 0,1 , or $\geq 2$ prior antibiotic exposures are shown in Fig. 2 and Table 1. Among all pathogens, a trend toward slightly higher incidences of fluoroquinolone nonsusceptibility and of multidrug-resistant and ESBL phenotypes wase seen with $\geq 2$ prior antibiotic exposures than with 1 or 0 (Fig. 2); however, with the exception of ESBL phenotype in patients with $\geq 2$ vs 1 prior antibiotic exposures $(6.6 \%$ vs $3.6 \%$; $95 \%$ CI $0.1,7.7 ; p=0.041)$, these differences in incidence were not statistically significant. This trend seemed to be driven largely by susceptibility pattern changes observed with $K$. pneumoniae (Table 1 ).

\section{Discussion}

The aim of this study was to characterize the distribution and antibiotic nonsusceptibility profiles of key Gram-negative organisms by the presence of antibiotic exposures among patients with hospital-onset UTIs. Although numerous patient factors can affect pathogen distribution and susceptibility trends, we focused on this one risk factor given that it is modifiable and easily evaluated by clinicians using the medical record. Antibiotic exposure is an important consideration given a recent multicenter prevalence study describing antibiotic use in $50 \%$ of inpatients on any given day during admission [11]. Therefore, our approach focusing on the number of exposures offers a simple and straightforward supplement to guide empirical antibiotic selection for hospitalonset UTI treatment.

Our study focusing on the single risk factor of antibiotic exposure produced several notable findings. Consistent with epidemiologic trends, E. coli (49.5\%) was the most commonly isolated pathogen $[8,9,12]$, and this was consistent across all categories of prior antibiotic exposure. Although the simple binary designation of prior antibiotic exposure did not seem to affect overall pathogen distribution or nonsusceptibility trends,

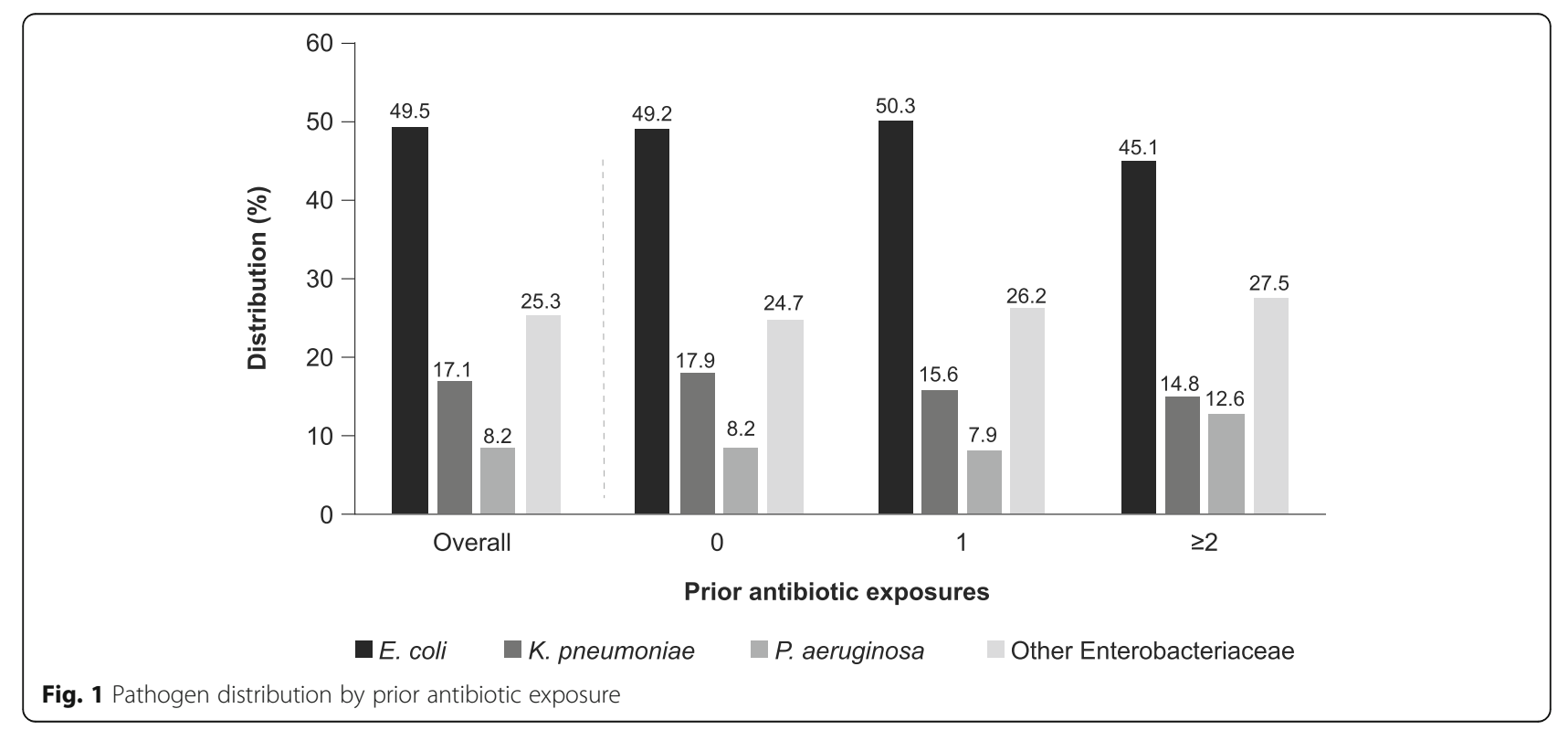




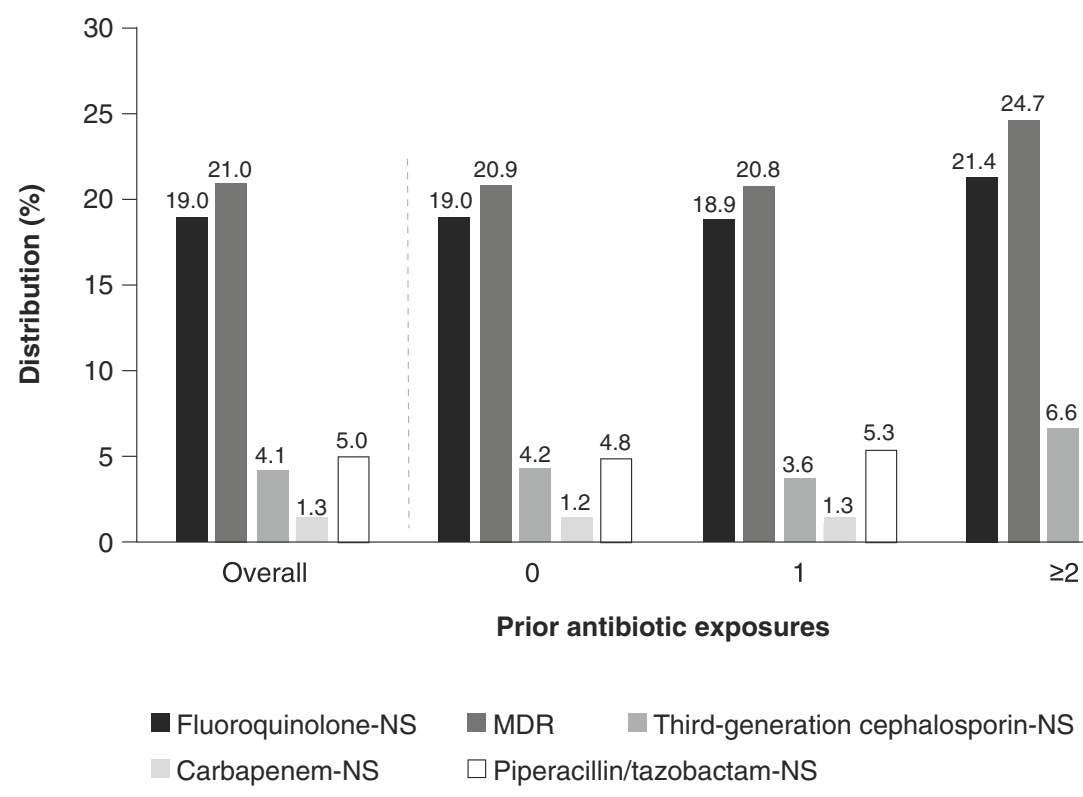

Fig. 2 Antibiotic nonsusceptibility phenotypes across all pathogens by prior antibiotic exposure. MDR multidrug resistant; NS nonsusceptible

differences became apparent on stratification by number of exposures. Prior receipt of $\geq 2$ antibiotic regimens was associated with a significantly higher frequency of $P$. aeruginosa UTIs and with slightly higher incidences of fluoroquinolone-, carbapenem-, and third-generation cephalosporin-nonsusceptibility as well as multidrug resistance. Collectively, our findings suggest that previous exposure to $\geq 2$ antibiotic regimens is associated with an alteration of the distribution and susceptibility profiles of Gram-negative pathogens among patients with hospital-onset UTIs.

Although antibiotic nonsusceptibility was highest among those with $\geq 2$ prior exposures to antibiotics, fluoroquinolone nonsusceptibility rates were $>18 \%$, even in the absence of prior antibiotic exposure. This finding is not unexpected given the large-scale use of fluoroquinolones for UTIs in both community and health care settings. Emergence of resistance to these antibiotics is recognized by a number of world health agencies and is considered a major public health concern $[1,2,12]$. Because practice guidelines recommend empirical use of fluoroquinolones for UTIs only if local drug resistance rates do not exceed 10\% [12], our findings suggest that caution should be exercised with empirical use of fluoroquinolones for hospital-onset UTIs, and strong consideration should be given to alternative therapies with broad-spectrum, Gram-negative coverage empirically.

Our findings also highlight the limitations associated with relying solely on antibiograms for patients with hospitalonset UTI, particularly those with $\geq 2$ antibiotic exposures. Although antibiograms are a useful starting point in the empirical drug selection process, they reflect the cumulative susceptibility rates for the first recovered pathogen in a particular patient, regardless of current site, timing of collection (community vs hospital onset), or patient-specific risk factors for resistance (eg, prior exposure to antibiotics) [7]. Recent guidelines from the Infectious Diseases Society of America and the Society for Hospital Epidemiology of America acknowledge several of these limitations and encourage the development of enhanced antibiograms stratified by various parameters (eg, age, location within the institution, infection site, patient comorbidities, and site of acquisition) to improve empirical selection of antibiotics. Furthermore, these guidelines encourage the development of institution-specific clinical treatment guidelines for common infectious diseases [13]. Our findings that prior antibiotic exposure alters the distribution of pathogens and the susceptibility profiles of common Gram-negative organisms implicated in UTIs occurring $\geq 3$ days after admission can easily be incorporated into clinical treatment guidelines to promote a more patient-specific approach to timely selection of appropriate empirical antibiotic therapy. In addition to guiding the selection of appropriately broad-spectrum empirical therapy for hospital-onset UTI, our findings may be helpful to institutional antimicrobial stewardship programs to target antibiotics likely to be inappropriate (eg, fluoroquinolones).

Some limitations should be considered when interpreting these findings. First, though we acknowledge that several factors may contribute to antibiotic-resistant infections, we purposefully did not assess multiple risk factors because data were not uniformly available for all patients. We also believed that inclusion of other patient factors would complicate our intent to create a simple, straightforward approach to guide empirical antibiotic 
Table 1 Nonsusceptibility phenotypes by prior antibiotic exposure

\begin{tabular}{|c|c|c|c|c|c|}
\hline \multirow[t]{2}{*}{ Pathogen } & \multirow[t]{2}{*}{ Nonsusceptibility phenotype } & \multirow{2}{*}{$\begin{array}{l}\text { Overall, \% NS } \\
(n / N)\end{array}$} & \multicolumn{3}{|c|}{ Prior antibiotic exposure } \\
\hline & & & 0 & 1 & $\geq 2$ \\
\hline \multirow[t]{5}{*}{ Escherichia coli $(n=3013)^{a, b}$} & Fluoroquinolone-NS & $25.4(758 / 2990)$ & $25.5(482 / 1888)$ & $24.8(253 / 1020)$ & $28.0(23 / 82)$ \\
\hline & Third-generation cephalosporin-NS & $3.5(105 / 3013)$ & $3.8(72 / 1903)$ & $2.7(28 / 1028)$ & $6.1(5 / 82)$ \\
\hline & MDR & $23.4(705 / 3013)$ & $24.1(458 / 1903)$ & $21.6(222 / 1028)$ & $30.5(25 / 82)$ \\
\hline & Carbapenem-NS & $0.1(4 / 3006)$ & $0.2(3 / 1900)$ & $0.1(1 / 1025)$ & $0(0 / 81)$ \\
\hline & Piperacillin/tazobactam-NS & $4.4(131 / 2965)$ & $4.6(86 / 1871)$ & $4.1(42 / 1014)$ & $3.8(3 / 80)$ \\
\hline \multirow[t]{5}{*}{ Klebsiella pneumoniae $(n=1039)^{\mathrm{a}, \mathrm{c}}$} & Fluoroquinolone-NS & $6.8(70 / 1036)$ & $6.0(41 / 689)$ & $7.5(24 / 320)$ & $18.5(5 / 27)$ \\
\hline & Third-generation cephalosporin-NS & $3.7(38 / 1039)$ & $2.7(19 / 692)$ & $5.0(16 / 320)$ & $11.1(3 / 27)$ \\
\hline & MDR & $10.1(105 / 1039)$ & $9.2(64 / 692)$ & $11.3(36 / 320)$ & $18.5(5 / 27)$ \\
\hline & Carbapenem-NS & $1.5(16 / 1035)$ & $1.0(7 / 690)$ & $2.5(8 / 319)$ & $3.8(1 / 26)$ \\
\hline & Piperacillin/tazobactam-NS & $7.1(73 / 1021)$ & $6.0(41 / 685)$ & $9.4(29 / 310)$ & $11.5(3 / 26)$ \\
\hline \multirow[t]{5}{*}{ Pseudomonas aeruginosa $(n=502)^{\mathrm{a}, \mathrm{d}}$} & Fluoroquinolone-NS & $21.8(109 / 499)$ & $22.2(70 / 316)$ & $20.6(33 / 160)$ & $26.1(6 / 23)$ \\
\hline & Third-generation cephalosporin-NS & $8.8(44 / 502)$ & $10.4(33 / 318)$ & $5.0(8 / 161)$ & $13.0(3 / 23)$ \\
\hline & MDR & $8.8(44 / 502)$ & $10.4(33 / 318)$ & $5.0(8 / 161)$ & $13.0(3 / 23)$ \\
\hline & Carbapenem-NS & $7.3(36 / 496)$ & $7.3(23 / 314)$ & $5.7(9 / 159)$ & $17.4(4 / 23)$ \\
\hline & Piperacillin/tazobactam-NS & $4.0(20 / 494)$ & $4.1(13 / 314)$ & $4.4(7 / 158)$ & $0(0 / 22)$ \\
\hline \multirow[t]{5}{*}{ Other Enterobacteriaceae $(n=1539)^{\mathrm{a}, \mathrm{e}}$} & Fluoroquinolone-NS & $14.1(216 / 1531)$ & $14.5(137 / 947)$ & $13.9(74 / 534)$ & $10.0(5 / 50)$ \\
\hline & Third-generation cephalosporin-NS & $3.9(60 / 1539)$ & $4.0(38 / 953)$ & $3.9(21 / 536)$ & $2.0(1 / 50)$ \\
\hline & MDR & $27.6(424 / 1539)$ & $26.4(252 / 953)$ & $29.9(160 / 536)$ & $24.0(12 / 50)$ \\
\hline & Carbapenem-NS & $1.5(23 / 1525)$ & $1.5(14 / 944)$ & $1.7(9 / 532)$ & $0(0 / 49)$ \\
\hline & Piperacillin/tazobactam-NS & $4.9(75 / 1529)$ & $4.8(45 / 947)$ & $5.4(29 / 533)$ & $2.0(1 / 49)$ \\
\hline
\end{tabular}

MDR multidrug resistant, $n$ number of nonsusceptible isolates, $N$ total number of isolates, $N S$ nonsusceptible

${ }^{a}$ For patients with missing susceptibility data, isolates were considered to be carbapenem-susceptible, piperacillin/tazobactam-susceptible, or non-MDR if they were susceptible to a third-generation cephalosporin. Otherwise, carbapenem, piperacillin/tazobactam, and MDR susceptibility data were considered missing, and isolates were not included in the analyses

${ }^{\mathrm{b}}$ E. coli: 903 isolates were missing carbapenem susceptibility data, of which 896 were classified according to the rules described above and seven were excluded from the analysis due to missing data; 1412 isolates were missing piperacillin/tazobactam susceptibility data, of which 1364 were classified according to the rules and 48 were excluded

${ }^{c}$ K. pneumoniae: 317 isolates were missing carbapenem susceptibility data, of which 313 were classified according to the rules and four were excluded; 443 isolates were missing piperacillin/tazobactam susceptibility data, of which 425 were classified according to the rules and 18 were excluded

${ }^{d} P$. aeruginosa: 97 isolates were missing carbapenem susceptibility data, of which 91 were classified according to the rules and six were excluded; 109 isolates were missing piperacillin/tazobactam susceptibility data, of which 101 were classified according to the rules and eight were excluded

eOther Enterobacteriaceae: 494 isolates were missing carbapenem susceptibility data, of which 480 were classified according to the rules and 14 were excluded;

721 isolates were missing piperacillin/tazobactam susceptibility data, of which 711 were classified according to the rules and ten were excluded

selection. Because we did not adjust for other factors, our findings cannot substantiate prior use of antibiotics as an independent risk factor for, or as a cause of, resistance, but our findings do show that prior use is an additional easily identifiable variable that can be used to guide empirical therapy. Second, the study was restricted to patients with UTIs occurring $\geq 3$ days after hospital admission. Although we had access to detailed hospitalization data, information on antibiotic and health care exposure before admission was limited. As such, we focused on patients for whom complete, detailed data were available. The 3-day time frame after hospital admission fails to account for recent antibiotic use in outpatient or other institutional settings; therefore, the potential effects of these exposures on our findings are unknown. Third, this study did not specifically quantify the relationship between cumulative duration of exposure to all or any given antibiotic and the presence of resistance. Given that the goal of the study was to provide clinicians with a straightforward, easily adaptable method for empirical antibiotic selection, we did not think it was necessary to specify with increased granularity the relationship between duration of prior antibiotic exposure and resistance. Although we were able to document prior receipt of many commonly used antibiotics with Gram-negative activity, data for other groups of antibiotics, including second-generation cephalosporins, and trimethoprim/sulfamethoxazole, were not available for this analysis. The relatively small number of patients with $\geq 2$ prior antibiotic exposures might also limit the interpretation of findings regarding resistance, though increased rates observed among fewer pathogens is still of concern. In addition, though the statistical analysis suggests some significant differences 
between patients with $\geq 2$ prior exposures and patients with 0 or 1 prior exposure, it should be noted that the numbers of patients in some of the groups were very small. As such, determination of the true statistical relevance of prior antibiotic exposure will require additional studies adequately powered to determine significance levels. Fourth, given that the presence of the ESBL phenotype among pathogens was not formally tested, the use of select third-generation cephalosporin resistance as a marker for ESBL may underestimate or overestimate the true incidence of ESBL. However, we believe third-generation cephalosporin resistance represented a practical surrogate because it is readily available to clinicians on urine culture susceptibility reports and that routine formal ESBL testing is not recommended by the Clinical and Laboratory Standards Institute (CLSI). Furthermore, given that culture susceptibility reports were from clinical and not reference laboratories, it can be assumed that third-generation cephalosporin resistance was reported using the US Food and Drug Administration-approved minimum inhibitory concentration breakpoint, which may better correlate with the presence of ESBL than CLSI breakpoints [14]. Finally, because findings are derived from positive urine cultures in the absence of a complete clinical picture, it is difficult to definitively distinguish between true infections and colonization. However, all patients received an antibiotic in response to a positive urine culture, which is suggestive of symptomatic infection for most patients.

\section{Conclusions}

Having received $\geq 2$ prior antibiotic regimens was associated with increased risk for a resistant Gram-negative pathogen among patients with hospital-onset UTIs. In addition to considering the hospital antibiogram, which should be stratified for greater usefulness, hospital clinicians should critically assess prior exposure to antibiotics when selecting empirical therapy for patients with UTIs that occur $\geq 3$ days after admission. Awareness of common pathogen distribution and nonsusceptibility trends among hospital patients with UTIs, combined with readily identifiable patient risk factors for infection with resistant pathogens, can provide a useful framework for clinicians during the empirical antibiotic selection process.

\section{Additional file}

Additional file 1: Patient demographics and hospital characteristics. (DOCX $13 \mathrm{~kb})$

\section{Abbreviations}

CLSI: Clinical and Laboratory Standards Institute; ESBL: Extended-spectrum $\beta$-lactamase; MDR: Multidrug resistant; NS: Nonsusceptible; UTI: Urinary tract infection

\section{Acknowledgments}

Editorial support for this manuscript was provided by Sally Mitchell, PhD, and Meher Dustoor, PhD, of ApotheCom, Yardley, PA, USA, and was funded by Merck \& Co., Inc., Kenilworth, NJ, USA.

Funding

This work was supported by Merck \& Co., Inc., Kenilworth, NJ, USA.

\section{Availability of data and materials}

The deidentified patient data that support the findings of this study were obtained from Premier Research Services, a division of Premier Inc.

(Charlotte, NC, USA). Restrictions apply to the availability of these data, which were used under license for the current study, and so are not publicly available. Data are, however, available from the authors upon reasonable request and with permission of Premier Inc.

\section{Authors' contributions}

MRB substantially contributed to the conception and design of the study, acquisition and analysis of data and interpretation of results, writing and critical review of the manuscript, gave approval of the final version of the manuscript to be published, and agreed to be accountable for the integrity of the data and the accuracy of the work. MPO substantially contributed to the conception and design of the study, acquisition and analysis of data and interpretation of results, writing and critical review of the manuscript, gave approval of the final version of the manuscript to be published, and agreed to be accountable for the integrity of the data and the accuracy of the work. MY substantially contributed to the conception and design of the study, acquisition and analysis of data and interpretation of results, writing and critical review of the manuscript, gave approval of the final version of the manuscript to be published, and agreed to be accountable for the integrity of the data and the accuracy of the work. JM substantially contributed to the conception and design of the study, acquisition and analysis of data and interpretation of results, writing and critical review of the manuscript, gave approval of the final version of the manuscript to be published, and agreed to be accountable for the integrity of the data and the accuracy of the work. TPL substantially contributed to the conception and design of the study, acquisition and analysis of data and interpretation of results, writing and critical review of the manuscript, gave approval of the final version of the manuscript to be published, and agreed to be accountable for the integrity of the data and the accuracy of the work. Employees of the study sponsor, in collaboration with the authors, were involved in the design, execution, analysis, and reporting of the research.

\section{Authors' information}

Not applicable.

\section{Competing interests}

MRB declares that she has no competing interests. MPO, MY, and JM were employees of Merck, Sharp \& Dohme Corp., a subsidiary of Merck \& Co., Inc., at the time of this study. TPL was a consultant for Merck, Sharp \& Dohme Corp., a subsidiary of Merck \& Co., Inc., at the time of the study.

Consent for publication

Not applicable.

\section{Ethics approval and consent to participate}

The patient data used in this study were purchased by Cubist Pharmaceuticals (subsequently acquired by Merck \& Co., Inc., Kenilworth, NJ, USA) from Premier Research Services, a division of Premier Inc. (Charlotte, NC, USA). In accordance with the Health Insurance Portability and Accountability Act (HIPAA) Privacy Rule, disclosed data from the Premier Healthcare Database were considered deidentified per 45 CFR 164.506(d)(2)(ii)(B) through the 'Expert Determination' method. In addition, use of these data was determined by Premier to be exempt from ethical approval under 45 CFR 46.101(b)(4), and this exemption was confirmed by the Western Institutional Review Board (Puyallup, WA, USA).

\section{Author details}

${ }^{1}$ Albany College of Pharmacy and Health Sciences, 106 New Scotland Avenue, Albany 12208-3492, NY, USA. ${ }^{2}$ Merck \& Co., Inc., Kenilworth, NJ, USA. 
Received: 23 September 2016 Accepted: 17 February 2017

\section{Published online: 28 February 2017}

\section{References}

1. World Health Organization. Antimicrobial resistance: global report on surveillance 2014. http://www.who.int/drugresistance/documents/ surveillancereport/en/. Accessed 16 Sept 2016.

2. Jones RN. Resistance patterns among nosocomial pathogens: trends over the past few years. Chest. 2001;119:397S-404.

3. Zilberberg MD, Shorr AF. Secular trends in Gram-negative resistance among urinary tract infection hospitalizations in the United States, 2000-2009. Infect Control Hosp Epidemiol. 2013;34:940-6.

4. Mauldin PD, Salgado CD, Hansen IS, Durup DT, Bosso JA. Attributable hospital cost and length of stay associated with health care-associated infections caused by antibiotic-resistant Gram-negative bacteria. Antimicrob Agents Chemother. 2010;54:109-15.

5. Thabit AK, Crandon JL, Nicolau DP. Antimicrobial resistance: impact on clinical and economic outcomes and the need for new antimicrobials. Exp Opin Pharmacother. 2015;16:159-77.

6. Peralta G, Lamelo M, Alvarez-Garcia P, Velasco M, Delgado A, Horcajada JP, Montero M, Roiz MP, Fariñas MC, Alonso J, Martínez LM, Gutiérrez-Macías A, Alava JA, Rodríguez A, Fleites A, Navarro V, Sirvent E, Capdevila JA, SemiBlee Study Group. Impact of empirical treatment in extended-spectrum beta-lactamase-producing Escherichia coli and Klebsiella spp. bacteremia: a multicentric cohort study. BMC Infect Dis. 2012;12:245.

7. Pakyz AL. The utility of hospital antibiograms as tools for guiding empiric therapy and tracking resistance: insights from the Society of Infectious Diseases Pharmacists. Pharmacotherapy. 2007;27:1306-12.

8. Hooton TM, Bradley SF, Cardenas DD, Colgan R, Geerlings SE, Rice JC, Saint S, Schaeffer AJ, Tambayh PA, Tenke P, Nicolle LE, Infectious Diseases Society of America. Diagnosis, prevention, and treatment of catheter-associated urinary tract infection in adults: 2009 International Clinical Practice Guidelines from the Infectious Diseases Society of America. Clin Infect Dis. 2010;50:625-63.

9. Metlay JP, Strom BL, Asch DA. Prior antimicrobial drug exposure: a risk factor for trimethoprim-sulfamethoxazole-resistant urinary tract infections. J Antimicrob Chemother. 2003;51:963-70.

10. Magiorakos AP, Srinivasan A, Carey RB, Carmeli Y, Falagas ME, Giske CG, Harbarth S, Hindler JF, Kahlmeter G, Olsson-Liljequist B, Paterson DL, Rice LB, Stelling J, Struelens MJ, Vatopoulos A, Weber JT, Monnet DL. Multidrugresistant, extensively drug-resistant and pandrug-resistant bacteria: an international expert proposal for interim standard definitions for acquired resistance. Clin Microbiol Infect. 2012;18:268-81.

11. Magill SS, Edwards JR, Beldavs ZG, Dumyati G, Janelle SJ, Kainer MA, Lynfield R, Nadle J, Neuhauser MM, Ray SM, Richards K, Rodriguez R, Thompson DL, Fridkin SK, Emerging Infections Program Healthcare-Associated Infections and Antimicrobial Use Prevalence Survey Team. Prevalence of antimicrobial use in US acute care hospitals, May-September 2011. JAMA. 2014;312:1438-46.

12. Gupta K, Hooton TM, Naber KG, Wullt B, Colgan R, Miller LG, Moran GJ, Nicolle LE, Raz R, Schaeffer AJ, Soper DE, Infectious Diseases Society of America; European Society for Microbiology and Infectious Diseases. International clinical practice guidelines for the treatment of acute uncomplicated cystitis and pyelonephritis in women: a 2010 update by the Infectious Diseases Society of America and the European Society for Microbiology and Infectious Diseases. Clin Infect Dis. 2011;52:e103-20.

13. Barlam TF, Cosgrove SE, Abbo LM, MacDougall C, Schuetz AN, Septimus E, Srinivasan A, Dellit TH, Falck-Ytter YT, Fishman NO, Hamilton CW, Jenkins TC, Lipsett PA, Malani PN, May LS, Moran GJ, Neuhauser MM, Newland JG, Ohl CA, Samore MH, Seo SK, Trivedi KK. Implementing an antibiotic stewardship program: guidelines by the Infectious Diseases Society of America and the Society for Healthcare Epidemiology of America. Clin Infect Dis. 2016;62:e51-77.

14. Mansury D, Motamedifar M, Sarvari J, Shirazi B, Khaledi A. Antibiotic susceptibility pattern and identification of extended spectrum beta-lactamases (ESBLs) in clinical isolates of Klebsiella pneumoniae from Shiraz, Iran. Iranian J Microbiol. 2016;8:55-61.

\section{Submit your next manuscript to BioMed Central and we will help you at every step:}

- We accept pre-submission inquiries

- Our selector tool helps you to find the most relevant journal

- We provide round the clock customer support

- Convenient online submission

- Thorough peer review

- Inclusion in PubMed and all major indexing services

- Maximum visibility for your research

Submit your manuscript at www.biomedcentral.com/submit
) Biomed Central 